Revue d'histoire de l'Amérique française

ZRS REVUE D.HISTOIRE DE L'AMÉRIQUE FRANÇAISE

\title{
Les Acadiens dans une seconde patrie : La Louisiane (suite)
}

Jeanne Grégoire

Volume 16, numéro 2, septembre 1962

URI : https://id.erudit.org/iderudit/302198ar

DOI : https://doi.org/10.7202/302198ar

Aller au sommaire du numéro

Éditeur(s)

Institut d'histoire de l'Amérique française

ISSN

0035-2357 (imprimé)

1492-1383 (numérique)

Découvrir la revue

Citer cet article

Grégoire, J. (1962). Les Acadiens dans une seconde patrie : La Louisiane (suite). Revue d'histoire de l'Amérique française, 16(2), 254-266.

https://doi.org/10.7202/302198ar d'utilisation que vous pouvez consulter en ligne.

https://apropos.erudit.org/fr/usagers/politique-dutilisation/ 


\title{
LES ACADIENS DANS UNE SECONDE PATRIE :
}

\author{
LA LOUISIANE * \\ (suite)
}

\section{MARIAGES (suite)}

Richard, Pierre, f. de Maximin et d'Isabelle Marguerite Landry, m. le 18 oct. 1794, avec Anna Patin, f. de Paul.

Rivera, Jean-Baptiste, de Genève, France, $f$. de Michel et de Rose Marin, m. le 20 mai 1794, avec Geneviève Fonteneau, f. de Jacques et de L. Doucet.

Robin, François, docteur en Droit civil et canonique, veuf de dame Clermont, m. le 15 avril 1793, avec Marie-Félicité Bellestre, f. de Louis Picoté.

Rougeau, Jean-Baptiste, f. de Firmin et d'Angélique Chartrand, m. le 15 janv. 1793, avec Eléonore Le Dé, f. de Louis et de Marie-Anne Trahan.

Roy, Augustin, f. d'Augustin et de Dorothée Gagné, m. le 6 oct. 1794, avec Victoire Cormier, f. de Michel et de Catherine.

Roy, Joseph, f. de Georges, m. nov. 1794, avec Euphrosine Carrière, $f$. de Jean.

St-Romain, Etienne, f. de Joseph et de Catherine Devaux, m. le 6 janv. 1794, avec Modeste Mayeux, f. de Joseph et d'Adrienne Bordelon.

Samson, Jean, f. de Pierre et de ... Guivode, m. le 17 janv. 1788, avec Agnès Vidrine, f. de Jean-Pierre de Vidrine et d'Isabelle Monchervaux.

Salien, Charles, $f$. de Michel et de Jeanne Monmarquier, m. le 31 janv. 1792, avec Angélique Fonteneau, f. d'Henri et de MarieLouise Doucel.

Saulnier, Sylvain, f. de Charles, m. mai 1789, avec Amélia Cornal. Savoy, François, f. de François, m. le 4 sept. 1792, avec MarieLouise Pitre, f. de Pierre et de Marie Bonnard.

Simar, Louis, du Canada, m. le 18 nov. 1788, avec Marie-Rose Trahan, f. de ... Maria Pons.

Sire, Jean, f. de Jean et de Barbara Waible, m. janv. 1792, avec Elisabeth Garret, f. de T. et de Mélanise Butchell.

* Voir notre Revue, XV : 572-593; XVI: 105-116. 
Smith, Charles, f. de Léonard et d'Isabelle Nil, m. le 30 avril 1792, avec Maria Santhif, f. de George et de Maria.

Stuts, Joseph, f. de Pierre et de Marie Miller, m. le 30 sept. 1792, avec Charlotte Banvillain, f. de Jacques.

Stuts, Michel, f. de Michel et d'Isabelle Stuts, m. le 22 mai 1787, avec Julienne Prud'homme, f. de Michel et de Catherine Raidnar.

Thibodeau, Pierre-Cyrille, f. de Pierre, m. le 15 juin 1790, avec Anna Adélaïde Chiasson, f. de Baptiste.

Trahan, Pierre, m. juin 1789, avec Pélagie S.

Vidrine, Pierre, f. de Jean-Pierre et d'Isabelle Monchervaux, m. le 7 juil. 1788, avec Marie-Berthe Brignac, f. de Jacques et de Marie Sylvestre.

Wale, Jean, f. de Jean, m. avril 1792, avec Marie-Louise Cavé.

\section{SEPULTURES}

Amaux, Maria Félonise, 14-9-1795

Anchet, (Mme), 16-7-1800

Anchet, Néré, 9-7-1791

Andrews, Jean (30 ans), 8-8-1803

Antrepont, Louis, 16-7-1803, José (fils de)

Ardois, François, 1790

Aucoin, Anastasie, 30-11-1802, ép. d'Amable Beaulieu

Aucoin, Perpétue (45 ans), 13-2-1805, ép. de N. Normand

Bailly, Lorenzo, 30-11-1800, f. de \& Laurence Bailly

Barré, Charles, 27-10-1779, f. de

Barré, (6 mois), f. de Charles

Barré, 4-6-1788, f. de Charles

Barré, Charlotte, 5-2-1803, veuve de Dutisme

Barré, Paul (enf.), 24-12-1782

Barret, Héléna, 19-2-1793

Baril, François, 28-11-1791

Bates, Anne, 6-11-1782, ép. de Guillaume Reed

Batin, Jean (1 an), 11-3-1783

Batone, François, 15-9-1802

Beaulieu, Fille (2 ans), 10-2-1783

Beaulieu, Amable-Bertrand, 27-9-1802, (de Canada)

Beaulieu, Céleste, 12-5-1802, f. de Marie

Beaulieu, François, 21-12-1793

Beaulieu, Jacob, 16-11-1796

Beaulieu, Pierre (enf.), 19-10-1792

Bélard, Antoine (63 ans), 12-2-1805, originaire de Picardie Belêtre (enf. 3 jrs), 10-10-1805, f. d'Antoine

Bello, Donat (5 m.), 9-11-1780 
Belle, Donat (le Sr), 20-12-1787

Belly, Jean (enf.), 20-1-1801

Benn, Jacob (60 ans), 4-11-1804

Benoit, Angèle, 1-11-1791

Benoit, Donatien, 24-8-1797

Benoit, Jean-Charles, 25-11-1787, f. de Jean-Charles

Benoit, Marie-Rose, 3-4-1801

Benoit, Olivier, 20-12-1787

Benoit, 5-2-1797, canadien, engagé de M. Despaux

Bernandez, (enf. 2 ans), 1-11-1802, f. de Michel

Bertrand, (2 ans), 19-8-1805, f. d'Amable

Bertrand, Nicolas (43 ans), 27-2-1804, ép. de Marguerite Pitre

Biles, Guillaume, 6-11-1803, f. de Jean et de Sarah

Bigras, Joseph (40 ans), 23-1-1805, célibataire

Blanchard, Maria, 19-10-1794

Boeuf, Charles (42 ans), 18-4-1783

Boisdoré, Antonio, 2-1794

Boisdoré, Eugénie, 28-8-1787

Boisdoré, Francoise, 1-12-1787

Boisdoré, Manet, 10-7-1793

Bondigne, (enf.), 31-8-1803, f. d'Adrien

Boon, Marie (20 m.) , 28-1-1804, f. de Daniel et de Anne Goudreau

Bordeleau (enf.), 7-6-1805, f. de Nicolas et de M. Dupuy

Boreys, Anne (28 ans), 12-10-1804, veuve d'Isaac Viennapon

Bossieu, Francois, 17-12-1780

Boulé, François, 7-6-1792

Boulet, Maria, 10-6-1788

Bourassa, Alexis, 10-8-1801, f. de Charles et de Madeleine Lalande

Bourassa, Charles, 10-10-1798

Bourassa, Charles, 1793

Bourassa, Gradenigo, 29-8-1801, f. de Charles et de Magdeleine Lalande

Bourassa, Louise (14 m.), 21-8-1780

Bourassa, Maria, 30-4-1786, f. de Jacques

Bourassa, Suzanne, 29-10-1799, f. de Charles

Bourdeaux, Bolacre, 11-9-1792

Bourg, Joseph (50 ans), 20-1-1806

Bourg, Marie-Louise $(6 \mathrm{~m}),. 16-10-1782$

Bourg, Lanze, 29-6-1788

Bourg, Lucie, 8-12-1795, f. de Jean et de Maria Richard

Bourg, Marie (13 ans), 11-8-1804, f. de Jean et de C. Richard

Bourg, Pierre, 28-10-1800, f. de

Boutin, François-Hyppolite, 15-10-1803, f. de Pierre et d'Anna Wilber

Boutin, Mme, 20-8-1788, ép. de Paul 
Boutin, Pierre, 25-2-1801

Braconnier, (enf.), 17-7-1802, f. d'Estevan

Brasseur, Angélique, 20-9-1789, f. de Nicolas

Bridon, André, 30-12-1787

Briginuo, Maria, 5-12-1799

Brignac, Jacques (4 ans), 8-10-1781

Brignac, Marie, 24-6-1801

Brignac, Marie, 7-5-1802, de Joseph Fonteneau

Broutin, Joseph, 26-11-1796

Broutin, Pierre, 2-9-1794

Brignac, Michel (40 ans), 9-12-1782

Brunet, François, 23-12-1792

Bureau, Jean, 20-5-1798

Burley, Cécile (23 ans), 21-5-1805, ép. de Jean-Baptiste Stelby

Burns, José (enf.), 5-8-1802, f. de Georges

Bushenel, (9 jrs), 8-9-1804

Buvogne, Elise, 18-12-1793

Cadieux, 23-9-1803, f. de Louis et de Marguerite Gradenigo

Caixt, Isabelle, 17-12-1797

Calahan, Daniel (enf.), 3-11-1787, f. de Daniel

Calahan (enf.), 20-12-1788, f. de Daniel

Callaghan, Jacinthe, 6-11-1796

Canmarsao, Maria, 15-7-1794

Caron, Joseph, 27-5-1789

Caron, Olivier, 30-12-1791, f. de

et de Marie-Jeanne Caron

Caron, Olivier, 4-1-1793

Carrière, (5 ans), 3-11-1803, f. de Michel et de M. Mercantel

Carrière, Josephe, 24-12-1787

Carrière (2 ans), 9-11-1803, f. de Michel et de M. Mercantel

Carrière, Joseph, 22-9-1795

Carrière, Joseph, 18-2-1790

Carteron (2 m.), 18-9-1805, f. de Nathaniel

Chiasson, Jean-Baptiste, 20-6-1788, un enf. de Jean-Baptiste

Chiasson (enf.), 31-12-1804, f. de M. Chiasson et de Marie Bourassa

Chiasson, Eloise, 5-1792

Casanova, Gertrude, 22-3-1802, f. de François et de Brigitte Roger

Cazaretti, Baptiste, 30-11-1795, (de la république de Grenova)

Chambon, Estevan, 15-2-1790

Chavot, Georges, 14-7-1790

Chremienque, (enf.), 20-12-1788, f. de Joseph

Cilet, (58 ans), 28-7-1780

Clavin, Marie-Louise, 4-1-1797

Clément, (enf.), 20-9-1803, f. de François 
Clouâtre, Marguerite, 6-4-1803, (engagé) ép. de François S

Clisson, Jean-Baptiste, 21-8-1788

Colin, Antoine, 28-8-1780, f. de Jean-Baptiste

Collins, Lucas, 20-6-1801

Collins, Marie (18 ans), 29-2-1804, f. de Jean

Collins, Marie

Collins, Sara, 15-6-1793

Comeau, (enf.), 6-7-1802, f. de Michel

Comeau, (fille), 14-2-1801, f. de Michel

Comeau, Charles (60 ans), 19-8-1805

Comeau, Emélie, 20-2-1801, f. de Michel et de Catherine Gagné Comeau, Irène, 1-12-1799, f. de et de Brown Comeau, Irène, 29-11-1799, f. de B. et de Cécile Langlois Cor, Barbara, 19-11-1800, f. de Michel Roman

Cormier, (enf.), 9-1-1806, f. d'Amable et de Modeste Richard

Cormier, Elise, 12-2-1799, f. d'Anaclet

Cormier, Joseph, 6-8-1795

Cormier, Maria, 2-11-1800

Cormier, Marianna, 7-7-1792

Cormier, Michel, 30-12-1790

Corriveau, (2 m.), 27-8-1805, f. de Nathaniel

Daniel, (2 ans), 19-9-1805, f. de Robert

Daniel, Louis, 12-1788

Daniel, Scistobar (2 ans), 19-9-1805

Debranle, Anne-Suzanne (enf.), 7-10-1802, f. de François et de Maria Carvu

Dejean, Maria, 5-2-1798, f. de Barthélémy

Delafosse, François

Delafosse, Roman, 17-11-1793

de la Mirande, Joseph (29 ans), 16-7-1780

de Nerbonne, Jean-Baptiste, 24-10-1792

de Soto, Manuel (Mme), 1-9-1799

Despaux, Guillaume, 12-12-1792, f. de Guillaume

Despaux, Rosine, 18-9-1794, f. de Guillaume

de Rochie, Alexandre, 5-1-1793

Desmarais, (enf.), 10-12-1788, f. de Georges

de Villars, Joséphine, 17-11-1793

de Villars, Dame Louis, 24-11-1793

de Villars, Richard, 22-11-1793

Deville, (enf. ondoyé), 22-6-1804, f. d'Etienne

Deville, (enf. 8 jrs), 27-8-1805, f. d'Etienne

D'Igliss, Pierre (45 ans), 30-12-1805

Donaguen, Marie (56 ans), 13-8-1780

Doucet, (18 ans), 21-2-1806

Doucet, Jean, 27-9-1803 
Doucet, Joseph, 25-12-1803

Doucet, Michel (105 ans), 13-10-1805

Doud, Roger (50 ans), 1804, irlandais

Dubuisson, Marie-Anne, 22-8-1788

Duclos, Catherine, 10- -1792

Ducrest, Armand (enf.), 13-11-1792, f. d'Armand

Dugas, Nanete, 16-6-1789

Dumoulin, Catherine, 17-12-1796

Duplantiers, Catherine (50 ans), 30-5-1804, ép. de Jean Tesson

Dupré, Jacques, 31-3-1799, f. de Santiago et de Théotiste Roy

Dupré dit Derbonne, Laurent (40 ans), 24-4-1783

Dupré, Louis (enf.), 24-10-1803, f. de Antonio et de Célestine Roy

Durand, Jean, 7-7-1790

Durocher, Louise, 10-7-1782, ép. de Jean-Baptiste Malavoye

Du Rousseau, (enf. 6 m.), 11-12-1803

$\mathrm{Du}$ Rougeau, Louis, 4-3-1800

Duverger, 12-7-1788

Emmanuel (métisse, 15 ans), 29-8-1801

Escoffier, François (50 ans), 5-3-1805

Faumeasque, (Mme Pedro), 6-9-1797

Favaron, Orsino, 4-10-1800, f. d'André

Frédéric, (enf.), 7-9-1789, f. de Joseph et d'Isabelle Roy

Félix, Baptiste (enf.), 2-6-1800

Félix, Jean-Baptiste, 9-7-1788, f. de Jean-Baptiste

Ferlan, Jacques, 28-10-1789

Fonteneau, (enf.), 22-10-1803, f. de François

Fonteneau, (enf. 5 m.), 25-9-1802, f. de José

Fonteneau, (20 ans), 30-8-1783

Fonteneau, (enf.), 23-9-1802, f. de Charles

Fonteneau, Baptiste (75 ans), 4-10-1805

Fonteneau, Claire (40 ans), 2-3-1804, ép. de Joseph Guillory

Fonteneau, Elise, 29-6-1793

Fonteneau, Mme François, 26-12-1791

Fonteneau, Henri, 19-9-1797

Fonteneau, Jacob, 6-8-1795

Fonteneau, Jean-Baptiste, 7-12-1801, ép. de Louisa Lagrange

Fonteneau, Julien, 1-2-1793

Fonteneau, Louise, 1793

Fonteneau, Luc, 10-12-1790

Fonteneau, Marguerite, 27-11-1801

Fonteneau, Marie-Divine, 6- -1792

Fonteneau, Michel, 25 janv. 1793

Fonteneau, Paul, 31-3-1799

Fonteneau, Santiago, 7-1-1801, f. de Santiago

Fonteneau, Velle, 15-10-1790 
Forest, (enf. 7 ans), 6-11-1803, f. de Pierre

Fost de l'Incarnation, 26-8-1790

Frugé, Eloise (10 ans), 19-12-1804, f. de Jean et de Eléonore...

Fragé, Pierre (60 ans), 20-8-1784

Gagnard, Jacques (60 ans), 1-1804

Gagné, Magdeleine, 19-4-1800, ép. de Joseph Chrétien

Ganet, Jean, 9-4-1801

Giroux, Pierre, 15-10-1803, f. de Pierre et de

Robert

Girouard, Marie, 15-1-1802, ép. de Michel Comeau

Golder, Michel, 29-12-1793

Gonor, Jean-Baptiste, 20-12-1787

Gomez, Jacques ( 77 ans), 8-2-1804, catalan d'origine

Gradenigo, Georges, 13-4-1801

Gradenigo, Jean, 11-12-1787, f. de Jean et de Marguerite Kress

Granger, Baptiste, 14-8-1795

Grenon, Emmanuel (40 ans), 14-3-1804

Guadatupe, Jean, 20-7-1787

Guédry, Baptiste, 14-2-1801, f. de David et de

Bordas

Guénard, Anastasie, 13-3-1789

Guénard, Joseph, 27-11-1787

Guiio, Pierre, 30-4-1789

Guilbris, (enf.) 15-4-1803, f. de Guillaume et de Marguerite Savoye

Guilbris, Joseph, 6-11-1802, f. de Guillaume et de Marguerite Savoye

Guillermont, Lydia, 12-3-1783

Guillori, Alexis, 10-7-1793

Guillori, Charles, 16-12-1792, f. de Joseph (nomade)

Guillori, Elise, 6-12-1795, f. de Pierre

Guillory, Pierre, 20-4-1803

Hattch, (enf.), 31-8-1803, f. de Samuel

Hébert, (enf., 2 ans), 8-10-1805, f. de Charles

Hébert, (enf.), 2-4-1803, f. de déf. Pierre

Heindsman, Magdeleine, 31-3-1803, engagée de Jean Tellers

Hinard, François, 23-6-1798

Ibis, Rocca, 9-10-1791

Irlandes, (Mr.), 4-1794

Jannis, Hubert, 10-11-1798

Jannis, José, 23-7-1802, f. de Michel

Jansone, Fébronie, 6-11-1799, f. de Jean

Jansone, Céleste, 18-7-1799, f. de Jean

Jannot, Jean-Baptiste, 10-10-1803, soltero de Jean-Baptiste, jr

Jannot, Ursule, 22-1-1804, ép. de Jean Gaignard

Labbé, Charlotte, 10-12-1787, engagé de M. François Lemelle

Lacasse, (enf.), 21-6-1803, f. de Charles 
Lacasse, Joseph (12 jrs), 8-9-1781

Lacasse, Marie (Mme), 21-6-1803, ép. de Louis Lavergne

Lacasse, Pierre, 24-12-1796, f. de Charles

Lachaisse, Louis, 14-12-1792

La Cour, Marguerite, 13-10-1796

La Fiolet, Modeste, 18-4-1797

Lafitte, Louise, 3-8-1798, f. de M. Lafitte

Lafleur, Baptiste, 10-5-1799

Lafleur, Cyprien, 29-12-1799, f. de Xavier

Lafleur, Diego (32 ans), 10-8-1804

Lafleur, Jacques (Mme), 1794

Lafleur, Marie-Jeanne, 21-7-1803, ép. de Joseph Moreau

Lalande, Geneviève, 10-12-1787, f. de Louis et d'Anastasie Beaulieu

Lallier, (enf.), 1-4-1802, f. de Joseph et de Marie-Jeanne Fonteneau

Lamarre, Maria Magdeleine, 12-1-1790

Lamirande, Joseph, 16-8-1796

Lamothe, Jacques, 25-12-1803, (vieux canadien)

L'Ancloux, Elise, 1798, f. de Baptiste

Landry, Eloise (4 ans), 24-4-1803, f. de Jean et d'Elise Bourg

Langevin, Charles, 3-12-1792, f. de Charles

Langlois, Didier, 4-5-1800,

Lassabe, Pierre (enf.), 15-6-1786, f. de Pierre Patrice

La Sonde, Cyprien, 3-11-1793

La Strape, Geneviève, 7-12-1797

Latiolet, Joseph, 21-4-1792

Lattier, Maria (enf.), 10-11-1801

Latulais, (1 an), 9-1-1783

Laurent, Marie-Claire, 28-10-1779, ép. de Guillaume Laurent

Lebrun, Thomas, 20-8-1793

Ledoux, Françoise, Mme, 3-3-1781, ép. de Fassieu

Ledoux, Michel, 6-10-1793

Léger, Jean (enf.), 1-1-1800

Léger, (enf.), 10-4-1788, f. de Louis et de Hyode

Léger, Michel, 8-2-1798, f. de Michel

Léger, Pierre, 10-11-1801, ép. de Maria Lottier

Lejeune, (enf. 2 ans), 12-10-1802, f. de José et de B. Maize

Lejeune, Marie, 27-2-1800

Lemelle, François, 18-9-1789

Le Mercier, Jean, 11-10-1798

Lemoine, Joseph, 6-12-1797

Lesassier, Julien, 22-12-1802, (capitaine)

Lesassier, Sarah (17 ans), 3-3-1804, ép. de Sr Gholson 
Lesassier, Suzanne (30 ans), 13-2-1804, f. de feu Julien et de Sarah Collins

Levye, Jean-Baptiste, 20-1-1789, ép. de Josephe Bourg

Liénard, Jean, 12-11-1792

Makoy, (enf. 2 m.), 20-11-1805, f. de Belly

Mallatte, Joseph (60 ans), 28-5-1805

Manuel, (60 ans), 10-1-1783

Marcatis, Denis (30 ans), 9-7-1780, natif de Karkan, Irlande (réfugié)

Mares, Joseph-Vincent, 23-8-1799

Margrier, Balthazar, 7-4-1790

Martin, (enf., 8 jrs), 9-6-1805, f. de Guillaume

Martin, Judith (50 ans), 29-1-1806, ép. de Remy Boudreau

Marie-Louise (créole, 30 ans), 1-3-1806, créole de Belêtre

Mariano, 14-1-1801

May, Santiago, 6-1-1788

Mayer, Agnès, 8-2-1792

Mendez, Amado, 24-2-1789

Mercantel, Julie, 27--1785, ép. de Louis Phéhon

Mercantel, Solasti, 16-1-1797

Milan, (Mme), 7-12-1795

Milan, Adélaïde, 5-9-1792

Milan, Félix, 18-10-1792

Milani, François, 13-1-1795, naturel de Pologne

Milet, Georges, 1785

Miller, Catherine, 11-2-1796

Miller, Cécile, 14-3-1799, f. de François

Miller, Georges, 1794

Miller, Manuel, 3-1-1798, f. de Frédéric

Miller, Marie, 25-5-1798

Mondou, André, 19-1-1796

Moreau, Augustin, 18-8-1794

Moreau, Clarisse (5 ans), 24-10-1803, f. d'Eustache et de Dorothée Roy

Moreau, Valentin (80 ans), 11-3-1782

Morin, Joseph (enf.), 19-9-1793

Morin, Léonard (17 ans), 27-1-1806, f. de Jean-Baptiste

Morisseau, Louis, -3-1792

Mulatrapa libre, Catherine, 19-12-1782

Mullins, Jacques, 7-1-1805, Irlandais d'origine

Nakin, Parricio, 11-7-1793

Navault, (enf.), 11-6-1804, f. d'André

Nezat, Antonio, 24-10-1795

Nezat, Antonio, 14-12-1796

Nezat, Antonio, 5-3-1798, f. d'Antonio 
Nezat, Eugénie, 24-9-1799, f. d'Antonio et de Tula Paré

Nolin, Claire (26 ans), 19-6-1780, ép. de Pierre Guidre

Normand, Charles (enf.), 21-12-1788, f. de Charles

Normand, Charles, 20-12-1791

Noto, Christophe, 18-1-1794, f. de Christophe.

Noto, Maria (enf.), 11-9-1800

O’Connor, Michel, 5-7-1800, f. de Pierre

Oguan, Claude, 13-8-1794

Olivier, Catherine, 10-6-1790

Ortega, Joseph, 20-10-1797, f. de Joseph

Ortolan, Zénon, 29-10-1801, f. de Jean et de Marie-Louise Vivarez

Ossot, Catherine, 10-3-1801

Paré, Adelcida, 14-10-1794

Patin, Marie-Marguerite, 29-10-1779

Patin, Marianna, 10-1-1795

Mozo Soltero Pedro, 23-6-1777

Perrault, Belisle, 6-12-1798

Perrault, Michel (Mme), 6-12-1798

Pierre, Sophia (enf.), 21-10-1793

Pitre, (enf.), 4-10-1804, f. de Dédé

Pitre, Euphrosine, 23-6-1777, f. de François

Pitre, Jacob, 18-9-1792

Pitre, Maria, 1797, f. d'Honoré

Pitre, Marie, 25-12-1794

Pitre, Paul (2 ans), 16-11-1780

Pitre, Pierre, 31-12-1794

Pitre, Thérèse, 18-10-1797

Pons, Joseph-André, 7-12-1795, f. de Joseph André et de Magdeleine Langlois

Préjean, Marin (1 jr), 24-4-1781

Poive, François, 1785

Primeau, Pierre, 10-8-1790

Proulx, Manuel, 21-11-1798

Prud'homme, Euphrosine (2 ans), 16-12-1783

Prud'homme, Julienne (35 ans), 5-2-1804, ép. d'Olivier Clark

Prud'homme, Marie, 6-3-1803, ép. d'Henri Hébert

Quercy, Noel, 10-9-1789

Raicraft, Georges, 30-7-1799

Ratier, Michel, 28-11-1795, f. de Michel et de Maria Stelly

Rayar, Jean (50 ans), 8-3-1789

Raynaud, Jean-Baptiste, 17-7-1788

Reed, Charlotte, 3-12-1802, ép. de Charles Baston (canadienne)

Reed, Guillaume (56 ans), 14-7-1804

Regnyder, Adélaide, 30-8-1802, f. d'Henri et de Marie Prud'homme 
Richard, Fabien (enf.), 30-4-1799, f. de Fabien

Richard, François, 9-2-1800, f. de François et de E. G.

Richard, Guillaume (20 ans)

Richard, Jean (7 ans), 27-2-1804, f. de Jean et de Isabelle N.

Richard, Louis, 23-8-1794

Richard, Louis (enf.), 26-2-1789, f. de Louis

Richard, Marguerite, 8-8-1795

Richard, Mathurin, 24-12-1796

Richard, Pierre (1 an), 4-7-1801, f. de Pierre et d'Anna Latricis

Rideaux, Jean, 30-9-1799

Ridoo, Juan, 12-1-1790

Ritters (enf.), 30-8-1803, f. de Jean

Ritters, Alexis, 30-12-1797

Rictex, Catherine, 12-4-1790

Ritter, Michel (55 ans), 5-3-1804

Rivard, François-Marie (61 ans), 1-7-1780

Rivest, Marie-Frégir, 19-6-1796

Robin, Mme, 1-11-1792

Rod, Noel, 2-1-1792

Roger, Louis (veuf), 50 ans, 7-2-1804

Rogiligné, Paul, 9-9-1800

Roman, Césarie, 13-10-1795

Romain, Désiré, 25-7-1790

Rosat, Barthélémy, 17-9-1787

Rosat, Jean, 17-7-1788

Rougeau, Marguerite, 28-11-1798

Roy, Auguste, 7-9-1800

Roy, Catherine, 13-9-1789

Roy, Céleste (18 ans), 3-11-1804, ép. d'Antoine Dupré

Roy, Joseph, 20-8-1793

Saint-Denis, Maria (Mme), 10-8-1797

St-Jean, Louis, 27-2-1798

Saint-Louis, 7-5-1789

Samson, (enf.), 6-8-1805, f. de Baptiste (ondoyé)

Samson, (enf.), 18-10-1802, f. de Baptiste et d'Isabelle Joubert

Samson, Baptiste, 8-12-1800, f. de Baptiste

Samson, Céleste (enf.), 16-3-1800

Samson, Charlotte (enf.), 13-3-1800

Samson, Hyppolite (12 ans), 23-1-1799, f. de Jean

Samson, Jean, 10-1-1793

Samson, Magdeleine (enf.), 5-3-1800

Samson, Maria (enf.), 16-3-1800

Saulnier, Etienne (2 ans), 24-8-1780

Saulnier, (enf., $10 \mathrm{jrs}$ ), 10-3-1806, f. de Sylvain et de Judith Bellot 
Saulnier, Silvinie, 25-1-1801

Saulnier, Sylvain, 2-1-1796

Saveyari, (Mme Charles), 29-4-1797

Savoye, Anna Maria (enf.), 14-10-1793

Savoye, Anastasie, 18-10-1802, ép. de Charles Comeau

Savoye, François (50 ans), 17-12-1780

Savoye, Jean, 13-10-1803

Savoye, Louise (20 m.), 12-11-1780

Savoye, Pierre, 4-3-1788

Sélassier, Angélique, 1789

Scita, (f. nat.), 6-10-1803

Snider, Jean, 2-4-1797

Snangervert, Magdeleine, 1794

Shahan, Thomas, 8-12-1801

Soileau, Marie, 7-10-1803, ép. d'Estevan Lamorandière, cap. de milice

Stelly, Baptiste, 3-4-1800, f. de Baptiste

Stelmays, Christine, 4-5-1802. veuve de George Stelly

Stelby, François (55 ans), 24-1-1806

Stelly, Georges, 23-7-1794

Stelly, Georges, 4-11-1792

Stelly, Thérèse, 16-12-1794

Stuts, Guillaume, 9-10-1794

Stuts, Michel, 13-12-1788

Stuts, Michel, 2-3-1803

Sylvestre, Maria, 8-8-1788, de Georges Desmarais

Tapasquiere, Joseph, 21-10-1794

Tessier, Joséphine (50 ans), 6-9-1804, sauvagesse, ép. de Jean Tessier

Thibodeau, Cyrille, 22-5-1801

Thibodeau, Marie (43 ans), 5-4-1803, ép. de Baptiste Chiasson

Thibodeau, Pierre, 20-8-1790

Thibodeau, Silésie, 28-8-1780

Thibodeau, Silésie (4 m.), 7-1-1781

Tison, Jean, 18-7-1795, f. de Jean

Trahan, Honoré, 10-7-1791

Trahan, Marie-Anne, 12-9-1797

Trahan, Marie-Jeanne, 3-1-1803, engagée de Balthazar Max

Trahan, Romain, 8-12-1788

Tevingue (enf.), 5-7-1803, f. de Daniel et de Geneviève Bam

Tilbrain, Marie-Jeanne, 21-7-1803, ép. de Paul Fonteneau

Truger, Nicolas, 19-12-1798

Vago (nat. Irl.), 9-12-1788

Valder, Antonio, 21-5-1792

Valet, Salomé, 9-2-1799, f. de Jean 
Vidrine, Elise (3 ans), 22-7-1803, f. de et de N. Soileau

Vidrine, Jean de (Senior), 6-1-1788

Vellevue, Henri, 31-1-1801, f. d'Henri

Veuillon, Benoni (50 ans), 29-10-1804

Waible (enf.), 3-8-1789, f. d'André

Waible, (enf.), 11-4-1803, f. de Jeanne

Waible, Joseph, 12-10-1793

Waible, Marie (9 m.), 2-8-1781

Waible, Michel (9 m.), 6-12-1783

Wamada, Astasia (enf.), 30-7-1803, f. nat. de Maria Vinta

Wamada, Janot, 23-10-1792, f. de Joseph

Woilhun, Jacob, 13-3-1789

White, Sarah, 11-12-1802, veuve de Lucas Collins

Il faut ajouter à ces sépultures, les suivantes:

un adulte, le 7-4-1783

Julie, 20-3-1783

M-Louise Sophie, 16-12-1780

Pour terminer l'année 1791, se trouve la liste suivante: un enfant de François Mercantel

$\begin{array}{lll}\text { “ “ } & \text { " Michel Lucas et de Picamio } \\ \text { “ “ } & \text { “ } & \text { Coan-Baptiste et de Erbonne } \\ \text { “ “ } & \text { “ } & \text { Collins } \\ \text { “ } & \text { " Anri Pargnèse }\end{array}$

Entre les dates du 14 mars et du 30 mai 1804 apparaît la note suivante:

«Pendant mon absence il a été enterré au cimetière de cette paroisse par le sacristain savoir:

Michel Comeau, 80 ans, chef de Sr Joseph Gradenigo

un enf. de Simon Fonteneau, la femme de ... Casemera, le

Sr Michel Ritter, une négresse de Jean Bourg, une négresse

de Charles Comeau, Mme Léonard, un enf. de Jean-Baptiste

Derbonne

Entre mai et juillet de l'année 1802, sans date précise, un enfant de Fabien Richard, un autre enfant Richard et trois nègres; un enfant Béland; un nègre de Michel Comeau; et Maya Balthazar ; un nègre de Julien Lapierre. »

$$
\text { (à suivre) }
$$

JEANNE GRÉGOIRE 